\section{Error-free replicative bypass of (6-4) photoproducts by DNA polymerase $\zeta$ in mouse and human cells}

\author{
Jung-Hoon Yoon, Louise Prakash, \\ and Satya Prakash ${ }^{1}$
}

Department of Biochemistry and Molecular Biology, University of Texas Medical Branch at Galveston, Galveston, Texas 77555 , USA

The ultraviolet (UV)-induced (6-4) pyrimidine-pyrimidone photoproduct $[(6-4)$ PP] confers a large structural distortion in DNA. Here we examine in human cells the roles of translesion synthesis (TLS) DNA polymerases (Pols) in promoting replication through a (6-4) TT photoproduct carried on a duplex plasmid where bidirectional replication initiates from an origin of replication. We show that TLS contributes to a large fraction of lesion bypass and that it is mostly error-free. We find that, whereas Pol $\eta$ and Pol ı provide alternate pathways for mutagenic TLS, surprisingly, Pol $\zeta$ functions independently of these Pols and in a predominantly error-free manner. We verify and extend these observations in mouse cells and conclude that, in human cells, TLS during replication can be markedly error-free even opposite a highly distorting DNA lesion.

Supplemental material is available at http://www.genesdev.org.

Received October 11, 2009; revised version accepted November 20, 2009.

Ultraviolet (UV) light induces the formation of two major photoproducts: cis-syn cyclobutane pyrimidine dimers (CPDs), and (6-4) pyrimidine-pyrimidone photoproducts [(6-4) PPs]. Whereas a CPD has a modest effect on DNA structure and does not significantly affect the ability of the two pyrimidines to form a Watson-Crick base pair with the correct purine base, a (6-4) PP induces a large structural distortion in DNA, as it confers a $44^{\circ}$ bend in the DNA helix and the $3^{\prime} \mathrm{T}$ is oriented perpendicular to the $5^{\prime} \mathrm{T}$ in the (6-4) TT photoproduct (Kemmink et al. 1987; Kim and Choi 1995; Kim et al. 1995; Lee et al. 1999). As a consequence, a (6-4) TT photoproduct is highly inhibitory to synthesis by yeast and human translesion synthesis (TLS) polymerases (Pols) (Prakash et al. 2005). Although both yeast and human Pol $\eta$ can insert a G opposite the $3^{\prime}$ T of the lesion, they do so with a much lower catalytic efficiency than for the insertion of an A opposite undamaged $\mathrm{T}$, and neither polymerase can carry out the subsequent extension step (Johnson et al. 2001). Human Pol can also incorporate a G or an A opposite the

[Keywords: Replicative lesion bypass; DNA replication; (6-4) photoproducts; UV damage; DNA repair]

${ }^{1}$ Corresponding author.

E-MAIL s.prakash@utmb.edu; FAX (409) 747-8610.

Article is online at http://www.genesdev.org/cgi/doi/10.1101/gad.1872810. $3^{\prime} \mathrm{T}$ of the photoproduct, but with a poor catalytic efficiency, and it also is unable to perform the further extension (Johnson et al. 2000; Haracska et al. 2001). Biochemical studies with Pol $\zeta$, which have been limited to the yeast protein because of the unavailability of purified human protein, have shown that it lacks the ability to insert a nucleotide opposite the $3^{\prime} \mathrm{T}$ of the lesion, but it can proficiently extend from the correct as well as the incorrect nucleotides inserted opposite the 3' T by another Pol (Johnson et al. 2000, 2001). Hence, TLS opposite a (6-4) TT photoproduct could be carried out by the sequential action of two Pols where, following the insertion of a nucleotide opposite the $3^{\prime} \mathrm{T}$ by Pol $\eta$ or Pol $\iota$, Pol $\zeta$ could perform the subsequent extension reaction.

The poor catalytic efficiency of Pol $\eta$ and Pol $\iota$ and their propensity for inserting an incorrect nucleotide, as well as the proficient ability of $\mathrm{Pol} \zeta$ to extend from the incorrect nucleotides, would suggest that TLS opposite a (6-4) PP will occur infrequently and be highly mutagenic. However, contrary to such expectations, we show here that, during replication in human cells, TLS can account for a very substantial fraction of lesion bypass and that it is markedly error-free. Moreover, from studies in both human and mouse cells, we provide evidence for the predominant role of Pol $\zeta$ in TLS opposite (6-4) PPs and show that it functions in TLS in a highly error-free manner, rather than in an error-prone manner predicted from in vitro biochemical analyses. Also, in sharp contrast to our observations that, during replication, TLS opposite (6-4) PPs occurs in a predominantly error-free fashion and that Pol $\zeta$ functions in TLS in a nonmutagenic manner in both mouse and human cells, TLS opposite this lesion in a gapped plasmid exhibits very high mutagenicity, with Pol $\zeta$ being responsible for this errorproneness (Shachar et al. 2009). We elaborate on the implications of these and other observations and conclude that mammalian cells have evolved the means via which TLS Pols can perform lesion bypass during replication in an effective and error-free manner, and this applies even if the DNA lesion is a highly distorting one as exemplified by (6-4) PP.

\section{Results and Discussion}

Here we examine TLS in human cells opposite a (6-4) TT PP carried on the leading or the lagging strand DNA template in a duplex plasmid where bidirectional replication initiates from a replication origin (Danna and Nathans 1972; Fareed et al. 1972; Li and Kelly 1985; Bullock et al. 1991) and that therefore allows for the analysis of TLS on both DNA strands (Supplemental Fig. 1). In this system, TLS through the lesion, regardless of whether it is error-free or mutagenic, would result in a blue colony on LB + X-gal/kan medium, whereas removal of the lesion by nucleotide excision repair (NER) or lesion bypass by template switching would produce a white colony. For all of the studies reported here, for analyzing the contributions of different TLS Pols in promoting replication through the (6-4) TT PP, we performed experiments both with undamaged plasmid carrying the normal TT sequence and with the damaged plasmid. And, as expected, whereas with the undamaged plasmid the 
frequency of blue colonies among total $\mathrm{Kan}^{+}$colonies was not altered with siRNA knockdowns of any of the Pols, with the damaged plasmid the frequency of blue colonies among $\mathrm{Kan}^{+}$colonies showed considerable reductions upon depletion of the Pols, indicative of their role in TLS.

Involvement of Pol $\eta$, Pol $\iota$, and Pol $\zeta$ in TLS opposite a (6-4) TT PP in human cells

To avoid the removal of the (6-4) TT PP, we used XPA human fibroblast (HF) cells and examined the effects of siRNA knockdowns of TLS Pols on the frequency and mutagenesis of TLS through this photoproduct. The high efficiency of siRNA knockdown was verified by RT-PCR and by Western analysis as described (Yoon et al. 2009). As shown in Table 1, in XPA cells treated with control (NC) siRNA, TLS occurs with a frequency of $\sim 37 \%$ when the lesion is carried on the leading strand template, whereas on the lagging strand TLS occurs somewhat less frequently at $\sim 28 \%(P \ll 0.005)$. The knockdown of either Pol $\eta$, Pol $\iota$, or Pol $\kappa$ did not affect TLS frequencies on either DNA strand, whereas the knockdown of the Rev3 catalytic subunit or the Rev7 accessory subunit of Pol $\zeta$ conferred an $\sim 50 \%$ reduction in TLS frequencies for either DNA strand (Table 1). Since biochemical studies have indicated a role for Pol $\eta$ and Pol $\iota$ at the insertion step opposite a (6-4) TT PP (Johnson et al. 2000, 2001; Haracska et al. 2001), we next determined if Pol $\eta$ and Pol c could provide alternate means of TLS opposite the lesion. In that case, the simultaneous knockdowns of Pol $\eta$ and Pol $\iota$ would confer a much greater inhibition of TLS than that from their individual knockdown. In accord with this, we found that depletion of both Pol $\eta$ and Pol $\iota$ causes an $\sim 30 \%$ reduction in TLS frequencies on both DNA strands $(P \ll 0.005)$ (Table 1$)$.

Since Pol $\eta$ and Pol $\iota$ can insert a nucleotide opposite the $3^{\prime} \mathrm{T}$ of a (6-4) TT PP and Pol $\zeta$ can carry out the subsequent extension reaction, TLS through the lesion could be performed by the sequential action of these Pols wherein, following the nucleotide insertion by either Pol $\eta$ or Pol $\iota$, Pol $\zeta$ would extend. In that case, depletion of both Pol $\eta$ and Pol $\iota$ or of all three Pols-Pol $\eta$, Pol $\iota$, and Pol $\zeta$-would incur no more defect in TLS than that seen upon the depletion of $\mathrm{Pol} \zeta$ alone. Alternatively, if Pol $\zeta$ were to act independently of Pol $\eta$ and Pol $\iota$ such that Pol $\eta$, Pol $\iota$, and Pol $\zeta$ provided three alternate pathways for TLS through the (6-4) TT PP, then depletion of all three Pols would confer a much greater reduction in TLS frequencies than that seen upon the simultaneous depletion of any two of the Pols. To distinguish between these two possibilities, we examined the effects of knockdowns of these TLS Pols in Pol $\eta$-defective XPV cells. TLS on the leading and the lagging strands in XPV cells occurred with a frequency of $\sim 19 \%$ and $16 \%$, respectively (Table 2). Compared with XPA cells where Pol $\eta$ has been depleted (Table 1), TLS occurs with a lowered frequency in XPV cells, which suggests that NER removes a considerable fraction of (6-4) PPs from the duplex plasmid. As shown in Table 2, whereas Pol $\kappa$ knockdown had no effect, Pol ı knockdown conferred an $\sim 40 \%$ reduction in TLS frequencies on both the DNA strands $(P \ll 0.005)$. The knockdown of Rev3 or Rev7 subunits of Pol $\zeta$ led to $>50 \%$ reduction in TLS frequencies for the lesion carried on either strand $(P \ll 0.001)$, and the simultaneous knockdown of Rev3 and Pol ı caused an $\sim 80 \%$ reduction in TLS $(P \ll 0.001)$. Since the knockdown of both Pol $\zeta$ and Pol $\iota$ in XPV cells confers a much larger reduction in TLS frequencies than the knockdown of either Pol alone (Table 2), and since the simultaneous knockdown of Pol $\eta$ and Pol $\iota$ imparts a larger reduction in TLS frequencies than that from the depletion of either Pol alone (Table 1), we conclude that Pol $\eta$, Pol $\iota$, and Pol $\zeta$ provide three alternate routes for TLS opposite a (6-4) TT PP.

\section{Mutational analyses of TLS products from human cells}

Next, we determined the mutagenicity of TLS through the (6-4) TT PP. Quite unexpectedly, we find that, on both the DNA strands, TLS occurs predominantly in an errorfree manner, as $<2 \%$ of the TLS events from XPA cells had mutational changes (Supplemental Table 1), and they resulted primarily from the insertion of a $\mathrm{G}$ or a $\mathrm{T}$ opposite the $3^{\prime} \mathrm{T}$ or the $5^{\prime} \mathrm{T}$ of (6-4) TT PP. Also, quite surprisingly, for TLS on both DNA strands, the depletion of Rev3 or Rev7 conferred a more than twofold increase in the frequency of mutagenic TLS (Supplemental Table 1), and a comparison of the pooled data for both DNA strands in XPA NC siRNA-treated control cells versus those transfected with Rev3 or Rev7 siRNA shows that the increase in mutation frequencies upon Pol $\zeta$ knockdown is statistically highly significant $(P \ll 0.005)$. Although we find no significant change in mutation frequencies or in mutational pattern upon the knockdown of Pol $\eta$ or Pol $\iota$ alone, the simultaneous knockdown of both of these Pols led to a complete absence of mutations resulting from TLS through the (6-4) TT PP carried on either of the DNA strands (Supplemental Table 1). To further verify the involvement of Pol $\eta$ and Pol $\iota$ in mutagenic TLS opposite the (6-4) TT PP, we compared the mutational frequencies and the types of nucleotides inserted during TLS in XPV

Table 1. Effects of siRNA knockdowns of Pol $\eta$, Pol $\iota$, Pol $\kappa$, and Pol $\zeta$ on TLS opposite a (6-4) TT photoproduct carried on the leading or lagging strand DNA template in NER-defective XPA HF cells

\begin{tabular}{|c|c|c|c|c|c|c|}
\hline \multirow[b]{2}{*}{ siRNA } & \multicolumn{3}{|c|}{ Leading strand } & \multicolumn{3}{|c|}{ Lagging strand } \\
\hline & $\begin{array}{l}\text { Number of } \\
\text { Kan }^{+} \text {colonies }\end{array}$ & $\begin{array}{c}\text { Number of blue } \\
\text { colonies among } \text { Kan }^{+}\end{array}$ & $\begin{array}{c}\text { TLS } \\
\text { percentage }\end{array}$ & $\begin{array}{l}\text { Number of } \\
\mathrm{Kan}^{+} \text {colonies }\end{array}$ & $\begin{array}{c}\text { Number of blue } \\
\text { colonies among } \text { Kan }^{+}\end{array}$ & $\begin{array}{c}\text { TLS } \\
\text { percentage }\end{array}$ \\
\hline $\mathrm{NC}$ & 673 & 252 & $37.4 \%$ & 456 & 132 & $27.9 \%$ \\
\hline Pol $\eta$ & 637 & 243 & $38.1 \%$ & 655 & 199 & $30.8 \%$ \\
\hline Pol Ł & 582 & 232 & $39.9 \%$ & 536 & 144 & $26.8 \%$ \\
\hline Pol к & 601 & 238 & $39.6 \%$ & 489 & 140 & $28.6 \%$ \\
\hline Rev3 & 606 & 111 & $18.3 \%$ & 550 & 76 & $13.8 \%$ \\
\hline Rev7 & 646 & 114 & $17.6 \%$ & 586 & 80 & $13.7 \%$ \\
\hline Pol $\eta+$ Pol $\iota$ & 802 & 220 & $27.4 \%$ & 641 & 130 & $20.3 \%$ \\
\hline
\end{tabular}


Table 2. Effects of siRNA knockdowns of Pol $\eta$, Pol $\iota$, Pol $\kappa$, and Pol $\zeta$ on TLS opposite a (6-4) TT photoproduct carried on the leading or lagging strand DNA template in Pol $\eta$-defective XPV (XP30RO) HF cells

\begin{tabular}{|c|c|c|c|c|c|c|}
\hline \multirow[b]{2}{*}{ siRNA } & \multicolumn{3}{|c|}{ Leading strand } & \multicolumn{3}{|c|}{ Lagging strand } \\
\hline & $\begin{array}{l}\text { Number of } \\
\mathrm{Kan}^{+} \text {colonies }\end{array}$ & $\begin{array}{c}\text { Number of blue } \\
\text { colonies among } \mathrm{Kan}^{+}\end{array}$ & TLS percentage & $\begin{array}{l}\text { Number of } \\
\mathrm{Kan}^{+} \text {colonies }\end{array}$ & $\begin{array}{c}\text { Number of blue } \\
\text { colonies among Kan }{ }^{+}\end{array}$ & $\begin{array}{c}\text { TLS } \\
\text { percentage }\end{array}$ \\
\hline NC & 482 & 90 & $18.6 \%$ & 542 & 89 & $16.3 \%$ \\
\hline Pol ı & 475 & 51 & $10.7 \%$ & 485 & 46 & $9.5 \%$ \\
\hline Pol к & 527 & 95 & $18.1 \%$ & 553 & 93 & $16.8 \%$ \\
\hline Rev3 & 584 & 51 & $8.7 \%$ & 564 & 42 & $7.4 \%$ \\
\hline Rev7 & 589 & 50 & $8.5 \%$ & 617 & 44 & $7.1 \%$ \\
\hline Rev3 + Pol ı & 515 & 19 & $3.7 \%$ & 493 & 17 & $3.4 \%$ \\
\hline
\end{tabular}

cells transfected with control siRNA versus those transfected with Pol ı siRNA. In contrast to XPV control cells where seven mutational events were recovered among the 518 TLS products analyzed for the two DNA strands, we found no mutagenic events among the almost 800 TLS products obtained from XPV cells that had been transfected with Pol ı siRNA (Supplemental Table 2). Thus, overall, these data indicated that, in contrast to Pol $\zeta$, which promotes error-free TLS opposite (6-4) TT PP, Pol $\eta$ and Pol $\iota$ contribute to mutagenic TLS through this lesion. However, because the need for sequencing limited the number of mutational events that could be analyzed with the plasmid system in human cells, we resorted to examining this question using mutational analyses for a gene carried in the mouse genome, where a much larger number of TLS events could be examined. As discussed below, from studies in mouse cells, we are able to verify and extend this conclusion to (6-4) PPs that form at various dipyrimidine sites.

Error-prone role of Pol $\eta$ and Pol $\iota$ and error-free role of Pol $\zeta$ in TLS through (6-4) PPS in mouse cells

Since, in addition to TT sites, (6-4) PPs form also at TC and CC sites, we next examined the effects of siRNA knockdowns of TLS Pols on UV-induced mutations in the cII transgene that has been integrated into the genome in a big blue mouse embryonic fibroblast (BBMEF) cell line (Jakubczak et al. 1996; You et al. 2001). The highly efficient knockdowns of Pols in mouse cells was verified as described (Yoon et al. 2009). In several previous studies, this system has been shown to exhibit similar mutational responses to DNA-damaging agents as those observed with endogenous chromosomal genes (You and Pfeifer 2001; You et al. 2001; Besaratinia and Pfeifer 2006). To ensure that UV mutations resulted specifically from TLS opposite (6-4) PPs, CPDs were selectively removed from the genome by expressing a CPD photolyase gene in BBMEF cells and using the experimental protocol that allows for the complete removal of CPDs (You et al. 2001).

As shown in Table 3, the frequency of spontaneous mutations in the $c I I$ gene in nonirradiated mouse cells treated with control (NC) siRNA and with or without exposure to photoreactivating light was $\sim 13 \times 10^{-5}$. As expected, in nonirradiated cells, siRNA knockdown of any of the TLS Pols-Pol $\eta$, Pol $\iota$, Pol $\kappa$, or Pol $\zeta$-or of both Pol $\eta$ and Pol $\iota$, had no effect on this spontaneous mutation frequency (Supplemental Table 3). In UV-irradiated $\left(5 \mathrm{~J} / \mathrm{m}^{2}\right)$ mouse cells expressing the CPD photolyase but not exposed to photoreactivating light, the $c I I$ mutation frequency rose to $\sim 53 \times 10^{-5}$, whereas upon photoreactivation, the mutation frequency declined to $\sim 25 \times 10^{-5}$ (Table 3). Since spontaneous mutations are included among these UV-induced mutation frequencies, subtraction of the spontaneous mutation frequency $\left(\sim 13 \times 10^{-5}\right)$

Table 3. UV-induced mutation frequencies in the cII gene in (BBMEF) mouse cells expressing a CPD photolyase and treated with siRNAs for different TLS Pols

\begin{tabular}{|c|c|c|c|c|}
\hline siRNA & $U^{\mathrm{a}}$ & Photoreactivation $^{\mathrm{b}}$ & $\begin{array}{l}\text { Mutation frequency } \\
\qquad\left(\times 10^{-5}\right)\end{array}$ & $\begin{array}{c}\text { Mutation frequency derived from TLS } \\
\text { opposite }(6-4) \mathrm{PPs}^{\mathrm{d}}\left(\times 10^{-5}\right)\end{array}$ \\
\hline $\mathrm{NC}^{\mathrm{e}}$ & - & - & $13.2 \pm 2.8^{\mathrm{f}}$ & \\
\hline $\mathrm{NC}$ & - & + & $12.8 \pm 2.4$ & \\
\hline $\mathrm{NC}$ & + & - & $52.7 \pm 3.8$ & \\
\hline $\mathrm{NC}$ & + & + & $25.4 \pm 3.6$ & 12.4 \\
\hline Pol $\eta$ & + & + & $18.6 \pm 2.8$ & 5.6 \\
\hline Pol ८ & + & + & $16.8 \pm 2.1$ & 3.8 \\
\hline Pol $\eta+$ Pol ı & + & + & $14.3 \pm 2.4$ & 1.3 \\
\hline Rev3 & + & + & $36.4 \pm 4.8$ & 23.4 \\
\hline
\end{tabular}

${ }^{\mathrm{a}}$ Five jules per square meter UVC (254 nm) light.

${ }^{\mathrm{b}}$ Photoreactivation with UVA (360 nm) light for $3 \mathrm{~h}$.

${ }^{\mathrm{c}}$ Mutation frequency data were obtained from averages of five to seven independent experiments.

${ }^{\mathrm{d}}$ These mutation frequencies have been obtained from subtracting spontaneous mutation frequencies $\left(13.0 \times 10^{-5}\right)$, and hence represent mutation frequencies that derive from TLS opposite (6-4) PPs.

e(NC) Negative control siRNA.

$\mathrm{f}( \pm)$ Indicates SD. 
yields a mutation frequency of $\sim 40 \times 10^{-5}$ that can be ascribed to resulting from TLS opposite both CPDs and (6-4) PPs. Since the removal of CPDs by photoreactivation yields a mutation frequency of $\sim 25 \times 10^{-5}$, subtraction of spontaneous frequencies yields a mutation frequency of $\sim 12 \times 10^{-5}$ (Table 3 ), which reflects mutations resulting from TLS opposite (6-4) PPs. Hence, we estimate that $\sim 30 \%$ of UV-induced mutations result from TLS opposite (6-4) PPs. Our estimate that exposure to UVC elicits the formation of $\sim 70 \%$ CPDs and $\sim 30 \%$ (6-4) PPs is supported also from studies in mouse cells where (6-4) PPs have been specifically removed by the expression of a (6-4) PP photolyase (Yoon et al. 2009). It is of interest to note that (6-4) PPs are induced more efficiently with lower-wavelength UVC irradiation than with higher-wavelength UVB irradiation (Yoon et al. 2000; You et al. 2001).

To analyze the roles of TLS Pols in the error-free versus error-prone bypass of (6-4) PPs, we depleted the Pol by siRNA treatment and determined the mutation frequencies in UV-irradiated mouse cells where CPDs have been removed by CPD photolyase following exposure to photoreactivating light. Whereas, as expected from TLS analyses in human cells, the knockdown of Pol $\kappa$ had no effect (data not shown), the knockdown of Pol $\eta$ or Pol $\iota$ either alone or together conferred a reduction in UVinduced mutation frequencies, and the knockdown of Rev3 led to an increase in mutation frequency (Table 3). Even though the depletion of either Pol $\eta$ or Pol $\iota$ alone had shown no reduction in the incidence of mutagenic TLS opposite (6-4) TT PP in human cells (Supplemental Table 1), depletion of either Pol confers a reduction in mutagenic TLS in mouse cells, where TLS could be examined opposite (6-4) PPs that form at the TC and $\mathrm{CC}$, as well as the TT sites. A comparison of mutation frequencies that can be attributed to resulting from TLS opposite (6-4) PPs in cells treated with control siRNA $\left(12.4 \times 10^{-5}\right)$ versus those treated simultaneously with Pol $\eta$ and Pol $\iota$ siRNA $\left(1.3 \times 10^{-5}\right)$ shows that, in cells depleted for both Pol $\eta$ and Pol $\iota$, the incidence of mutagenic TLS opposite (6-4) PPs declines so precipitously that the frequency of UV-induced mutations becomes almost the same as the spontaneous mutation frequencies observed in nonirradiated cells (Table 3). Depletion of the Rev3 subunit of Pol $\zeta$, on the other hand, leads to an approximately twofold increase in mutation frequencies (from $12.4 \times$ $10^{-5}$ to $23.4 \times 10^{-5}$ ) resulting from (6-4) PP bypass (Table 3). Our observations that, upon depletion of both Pol $\eta$ and Pol $\iota$, the error-free mode of TLS prevails, whereas upon depletion of Pol $\zeta$, the mutagenic mode of TLS becomes more prevalent, provide strong support for a highly errorfree role of Pol $\zeta$ in TLS opposite (6-4) PPs.

In Supplemental Figure 2, we show the mutational spectra resulting from TLS opposite (6-4) PPs in the cII gene following the knockdown of different TLS Pols. In mouse cells treated with control (NC) siRNA, mutational hot spots are located primarily at five dipyrimidine sequences (\#1-\#5) and, with the exception of hot spot \#4, they mostly incur $\mathrm{C} \rightarrow \mathrm{T}$ transition mutations. Although the frequency of UV-induced mutations resulting from (6-4) PP bypass rises upon Rev3 depletion (Table 3), the pattern of UV-induced mutations remains similar to that in control cells (Supplemental Fig. 2A). Interestingly, the knockdown of Pol $\eta$ versus Pol ı produces a distinct pattern of mutations, as the UV-induced mutational hot spots \#1 and \#4 are absent in cells depleted for Pol $\eta$ and hot spots \#2, \#3, and \#5 are absent in cells depleted for Pol ^ (Supplemental Fig. 2B). Most importantly, none of the UV-induced mutational hot spots remain in cells that have been depleted for both Pol $\eta$ and Pol $\iota$, and the mutational pattern becomes similar to that in nonirradiated control cells (Supplemental Fig. 2C). Since in cells depleted for both Pol $\eta$ and Pol TLS would occur via the Pol $\zeta$ pathway, this observation provides further support for a highly error-free role of Pol $\zeta$ in TLS opposite (6-4) PPs.

\section{Predominantly error-free replicative bypass of (6-4) PPs in human cells}

We conclude from these studies that, in human cells, TLS makes a prominent contribution to the replicative bypass of a (6-4) TT photoproduct, contributing to as much as $30 \%-40 \%$ of lesion bypass. Moreover, TLS opposite the photoproduct occurs in a predominantly error-free fashion, as $>98 \%$ of TLS events incur no mutations. Furthermore, we provide evidence that Pol $\zeta$ plays an important role in TLS opposite this lesion and, quite unexpectedly, it carries out this role in an error-free manner in human cells. Additional support for the error-free role of $\mathrm{Pol} \zeta$ is indicated from the analysis of UV mutagenesis originating from TLS opposite (6-4) photoproducts in mouse cells. In particular, our observation that, upon depletion of both Pol $\eta$ and Pol $\iota$, the frequency of UV-induced mutations that result from TLS opposite (6-4) PPs declines almost to the level of spontaneous mutation frequencies provides strong evidence for the error-free role of Pol $\zeta$. This conclusion is further reinforced by the very similar pattern of mutations in UV-irradiated mouse cells depleted of both Pol $\eta$ and Pol $\iota$ as that in nonirradiated cells.

The replicative bypass of (6-4) TT PPs by TLS Pols in human cells differs in many respects from that in yeast. Whereas in human cells TLS opposite this lesion makes a prominent contribution to lesion bypass and occurs predominantly in an error-free fashion, in yeast, TLS makes a rather small contribution and is highly mutagenic, as TLS could account for only $\sim 4 \%$ of lesion bypass, of which $\sim 60 \%$ incurred mutations (Bresson and Fuchs 2002). Also, it would appear that the TLS Pol $\eta$ and Pol $\zeta$ function in a more error-prone manner in yeast than in humans, since whereas in yeast TLS opposite a (6-4) TT PP would occur by the insertion of a G opposite the 3' T by Pol $\eta$ followed by extension by Pol $\zeta$ (Johnson et al. 2001; Bresson and Fuchs 2002; Prakash et al. 2005), in humans Pol $\zeta$ functions independently of Pol $\eta$ and promotes an error-free mode of TLS, and TLS by human Pol $\eta$ incurs only low-level mutagenesis.

\section{Model for pathways of replicative bypass of (6-4) PPs in human and mouse cells}

Based on the observations reported here and the available biochemical information for the role of yeast and human Pols in TLS opposite a (6-4) TT PP, we propose a scheme for TLS opposite this lesion in human and other mammalian cells (Fig. 1). In this scheme, Pol $\eta$, Pol $\iota$, and Pol $\zeta$ provide three alternate routes of replication through the lesion, in which Pol $\eta$ and Pol $\iota$ function in an error-prone manner and Pol $\zeta$ promotes error-free bypass. Since Pol $\eta$ and Pol $\iota$ can insert both the correct and incorrect 


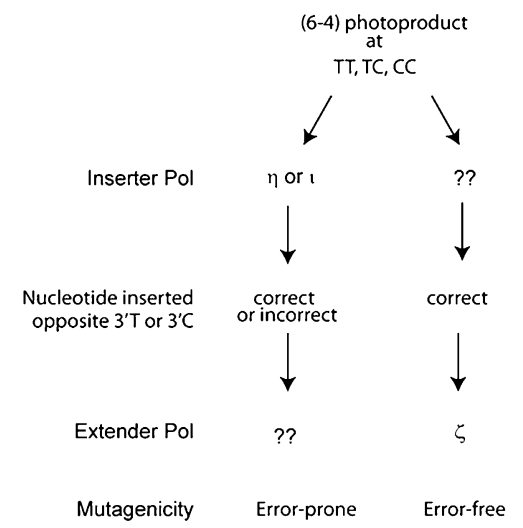

Figure 1. Pathways for replicative bypass of $(6-4)$ photoproducts in human and mouse cells. Pol $\eta$, Pol $\iota$, and Pol $\zeta$ provide three alternate routes of TLS. Following nucleotide insertion opposite the $3^{\prime} \mathrm{T}$ or 3' C of the (6-4) photoproduct by either Pol $\eta$ or by Pol $\iota$, an as-yetunidentified Pol would extend, and TLS by these Pols is primarily responsible for the mutational events. In Pol $\zeta$-mediated TLS, following nucleotide insertion opposite the $3^{\prime} \mathrm{T}$ or $3^{\prime} \mathrm{C}$ by a Pol that remains to be identified, Pol $\zeta$ would carry out the extension reaction, and this pathway is largely error-free.

nucleotides opposite the $3^{\prime} \mathrm{T}$ of the photoproduct but are unable to carry out the subsequent extension (Johnson et al. 2000, 2001; Haracska et al. 2001), we suggest the involvement of an as-yet-unidentified DNA polymerase at the extension step. From the proficient ability of yeast Pol $\zeta$ to carry out the extension reaction and its inability to perform the insertion reaction opposite the $3^{\prime} \mathrm{T}$ (Johnson et al. 2000, 2001), we assume that mammalian Pol $\zeta$ also functions only at the extension step, and hence we suggest that an as-yet-unidentified polymerase carries out the insertion opposite the $3^{\prime} \mathrm{T}$ or $3^{\prime} \mathrm{C}$ of a (6-4) PP from which Pol $\zeta$ then extends. An important corollary of this model is that Pol $\eta$ or Pol $\iota$ function together with an extender polymerase that is different from Pol $\zeta$, and that Pol $\zeta$ functions with an inserter polymerase other than Pol $\eta$ or Pol ı. The identity of such an inserter and extender Pol remains unknown at present, and whether the low-fidelity Pols such as Pol $\beta$, Pol $\lambda$, or any other, function at these steps remains to be determined. Also, it would be of considerable interest to know how such a distinct set of inserter and extender Pols as proposed here (Fig. 1) coordinate their actions in lesion bypass, and to unravel how Pol $\zeta$-dependent TLS attains the high degree of accuracy opposite a distorting lesion such as a (6-4) PP.

\section{TLS during replication differs from that occurring during a gap-filling reaction}

A comparison of our results for TLS opposite the (6-4) TT photoproduct carried in a duplex plasmid with those where TLS opposite this lesion was examined in a gapped plasmid reveals striking differences in the way TLS operates in human cells during replication versus during the gap-filling reaction. In contrast to our observations, which indicate a highly error-free mode of TLS opposite the (6-4) TT PP $(\ll 2 \%$ errors) regardless of whether TLS occurred during the replication of the leading or the lagging DNA strand, in the gapped plasmid, TLS across this lesion was found to occur in such a highly mutagenic fashion that $\sim 75 \%$ of the TLS events were mutagenic
(Shachar et al. 2009). Moreover, among the mutational events, while $\sim 50 \%$ occurred opposite the $3^{\prime} \mathrm{T}$ or the $5^{\prime} \mathrm{T}$ of the TT photoproduct, another $\sim 40 \%$ occurred at the nearest base flanking the $3^{\prime} \mathrm{T}$ or the $5^{\prime} \mathrm{T}$ of the lesion, and the remaining $\sim 10 \%$ involved deletions and insertions. In contrast, almost all of the mutations that resulted from TLS during replication of the duplex plasmid were restricted to sites opposite the $3^{\prime} \mathrm{T}$ or the $5^{\prime} \mathrm{T}$ of the photoproduct and incurred a base change. Furthermore, whereas we show that, during replication of the duplex plasmid, Pol $\zeta$ carries out error-free TLS opposite (6-4) TT PPs in human cells, and that it acts in a similar error-free fashion opposite (6-4) PPs formed at TT, TC, and CC sites in the mouse chromosomal cII gene, TLS studies with the gapped plasmid in mouse cells have indicated a highly error-prone role for Pol $\zeta$ opposite (6-4) TT PP such that almost no mutagenic TLS events are recovered in the absence of Pol $\zeta$ (Shachar et al. 2009). Hence, whereas during replication in human and mouse cells, TLS opposite the (6-4) PPs occurs in a predominantly error-free way and Pol $\zeta$ makes a very prominent contribution to it, during the gap-filling reaction, TLS occurs in a very highly mutagenic manner, with Pol $\zeta$ being primarily responsible for the error-prone mode. We conclude from this comparison that, in mouse and human cells, TLS during replication differs in fundamental ways from TLS that occurs during repair synthesis or other gap-filling reactions, and our observations point to the existence of regulatory mechanisms for promoting proficient and relatively error-free TLS during replication (see below).

\section{Conclusions and implications}

Although biochemical studies with yeast and human Pol $\eta$, Pol $\iota$, and Pol $\zeta$ have indicated that TLS opposite a (6-4) TT PP would occur infrequently and be highly mutagenic, we show that, during replication in human cells, TLS even opposite such a highly distorting lesion can account for a very significant fraction of lesion bypass and, moreover, it occurs in quite an error-free manner $(<2 \%$ errors). Also quite unexpected is our observation that, in both human and mouse cells, Pol $\zeta$ functions in TLS independently of Pol $\eta$ and Pol $\iota$, which are able to carry out the insertion reaction opposite the $3^{\prime} \mathrm{T}$ of the photoproduct; moreover, rather than extending from the nucleotides inserted by these Pols and contributing to mutagenic lesion bypass, as would have been expected from its proficient ability to extend from the correct as well as the incorrect nucleotides, Pol $\zeta$ promotes errorfree TLS. All of these observations, plus the fact that TLS during replication differs in fundamental ways from that occurring during a gap-filling reaction, lead us to suggest that human and other mammalian cells have evolved the means via which they have adapted TLS to play a major role in lesion bypass and to be quite error-free. Clearly, a strategy for replicating through the DNA lesions in an efficient and accurate manner would be much less disruptive to the rapid progression of the replication fork, and that would also curtail the risk of any chromosome rearrangements being generated. Since lesion bypass processes other than TLS would require template switching, such mechanisms, whether recombinational or nonrecombinational, would be much more disruptive to the progression of the replication fork and to the timely completion of S phase. Also, they pose the attendant risk of increased incidence of chromosome rearrangements 
resulting from the inadvertent pairing of related sequences present at nonhomologous sites.

Since, during replication, TLS Pols can bypass a (6-4) TT PP in quite an error-free fashion, we propose that, in human and other mammalian cells, TLS Pols function in collaboration with protein factors that not only coordinate the actions of different sets of inserter/extender TLS Pols with the replication ensemble, but that also modulate their efficiency and fidelity in lesion bypass. The elucidation of these mechanisms and of the mechanisms that enable an intrinsically low-fidelity Pol such as Pol $\zeta$ (Johnson et al. 2000; Prakash et al. 2005) to carry out predominantly error-free TLS during replication will require an in-depth understanding of the various components of the TLS machinery and of their functional interactions with the replisome. Also important will be the elucidation of possible regulatory mechanisms that affect the efficiency and fidelity of lesion bypass by the TLS Pols.

\section{Materials and methods}

For TLS studies in human cells, a (6-4) TT PP was carried on the leading or the lagging strand of a duplex plasmid where bidirectional replication initiates from an SV40 replication origin (see Supplemental Fig. 1). The detailed methods for the construction of the plasmid and for TLS studies in human cells with the duplex plasmid system have been described previously (Yoon et al. 2009). Briefly, high-proficiency depletion of TLS Pols from HF cells was achieved by siRNA treatments, the frequencies of TLS were determined from the number of blue colonies among total colonies that grew on LB + kan plates, and the mutational changes incorporated during TLS were analyzed for plasmid DNAs obtained from blue colonies (see Supplemental Fig. 1). For studies in mouse cells, we determined the frequencies of UV-induced mutations that would result specifically from TLS opposite (6-4) PPs formed at TT, TC, and CC sites. Mutation frequencies and mutational spectra were examined in the $c I I$ gene carried in BBMEF mouse cells expressing either the CPD photolyase or the neo-vector control (You et al. 2001), and highly efficient depletion of various TLS Pols was achieved by siRNA treatments as described (Yoon et al. 2009). The error-free or error-prone roles of Pols in TLS opposite (6-4) PPs were determined in UV-irradiated $\left(5 \mathrm{~J} / \mathrm{m}^{2}\right)$ BBMEF cells where the TLS Pol levels have been depleted by siRNA treatments and CPDs have been removed by the action of CPD photolyase in cells exposed to photoreactivating light for $3 \mathrm{~h}$ as described (You et al. 2001; Yoon et al. 2009). The detailed description of methods for TLS analysis in mouse and human cells have been described previously (Yoon et al. 2009).

\section{Acknowledgments}

We are grateful to Drs. James Cleaver (University of California at San Francisco, CA) and Gerd Pfeifer (Beckman Research Institute of the City of Hope, Duarte, CA) for providing the various SV40-transformed human cell lines, and to Dr. Thomas Wood, Director of the Recombinant DNA Laboratory, for sequencing the various TLS products generated in these studies. This work was supported by National Institute of Environmental Health Sciences (NIEHS) grant ES012411 and by NCI training grant T32CA117834 to J.H.Y. The (6-4) TT photoproduct-containing DNAs were constructed in the Synthetic Organic Chemistry Core Laboratory at University of Texas Medical Branch, supported by NIEHS Center grant P30-ES06676, and we are grateful to Dr. Richard Hodge for providing these DNAs.

\section{References}

Besaratinia A, Pfeifer GP. 2006. Investigating human cancer etiology by DNA lesion footprinting and mutagenicity analysis. Carcinogenesis 27: $1526-1537$.

Bresson A, Fuchs RPP. 2002. Lesion bypass in yeast cells: Pol $\eta$ participates in a multi-DNA polymerase process. EMBO J 21: 38813887.
Bullock PA, Seo YS, Hurwitz J. 1991. Initiation of simian virus 40 DNA synthesis in vitro. Mol Cell Biol 11: 2350-2361.

Danna KJ, Nathans D. 1972. Bidirectional replication of simian virus 40 DNA. Proc Natl Acad Sci 69: 3097-3100.

Fareed GC, Garon CF, Salzman NP. 1972. Origin and direction of simian virus 40 deoxyribonucleic acid replication. J Virol 10: 484-491.

Haracska L, Johnson RE, Unk I, Phillips BB, Hurwitz J, Prakash L, Prakash S. 2001. Targeting of human DNA polymerase $\iota$ to the replication machinery via interaction with PCNA. Proc Natl Acad Sci 98: 1425614261.

Jakubczak JL, Merlino G, French JE, Muller WJ, Paul B, Adhya S, Garges S. 1996. Analysis of genetic instability during mammary tumor progression using a novel selection-based assay for in vivo mutations in a bacteriophage $\lambda$ transgene target. Proc Natl Acad Sci 93: 90739078.

Johnson RE, Washington MT, Haracska L, Prakash S, Prakash L. 2000. Eukaryotic polymerases $\iota$ and $\zeta$ act sequentially to bypass DNA lesions. Nature 406: 1015-1019.

Johnson RE, Haracska L, Prakash S, Prakash L. 2001. Role of DNA polymerase $\eta$ in the bypass of a (6-4) TT photoproduct. Mol Cell Biol 21: 3558-3563.

Kemmink J, Boelens R, Koning T, van der Marel GA, van Boom JH, Kaptein R. 1987. ${ }^{1} \mathrm{H}$ NMR study of the exchangeable protons of the duplex d(GCGTTGCG).d(CGCAACGC) containing a thymine photodimer. Nucleic Acids Res 15: 4645-4653.

Kim J-K, Choi B-S. 1995. The solution structure of DNA duplex-decamer containing the $(6-4)$ photoproduct of thymidyl $\left(3^{\prime} \rightarrow 5^{\prime}\right)$ thymidine by NMR and relaxation matrix refinement. Eur J Biochem 228: 849-854.

Kim J-K, Patel D, Choi B-S. 1995. Contrasting structural impacts induced by cis-syn cyclobutane dimer and (6-4) adduct in DNA duplex decamers: Implication in mutagenesis and repair activity. Photochem Photobiol 62: 44-50.

Lee J-H, Hwang G-S, Choi B-S. 1999. Solution structure of a DNA decamer duplex containing the stable $3^{\prime}$ T.G base pair of the pyrimidine(6-4)pyrimidone photoproduct [(6-4) adduct]: Implications for the highly specific $3^{\prime} \mathrm{T} \rightarrow \mathrm{C}$ transition of the (6-4) adduct. Proc Natl Acad Sci 96: 6632-6636.

Li J, Kelly TJ. 1985. Simian virus 40 DNA replication in vitro: Specificity of initiation and evidence for bidirectional replication. Mol Cell Biol 5: $1238-1246$.

Prakash S, Johnson RE, Prakash L. 2005. Eukaryotic translesion synthesis DNA polymerases: Specificity of structure and function. Annu Rev Biochem 74: 317-353.

Shachar S, Ziv O, Avkin S, Adar S, Wittschieben J, Reißner T, Chanev S, Friedberg EC, Wang Z, Carell T, et al. 2009. Two-polymerase mechanisms dictate error-free and error-prone translesion DNA synthesis in mammals. EMBO I 28: 383-393.

Yoon J-H, Lee CS, O'Connor TR, Yasui A, Pfeifer GP. 2000. The DNA damage spectrum produced by simulated sunlight. $J$ Mol Biol 299: 681-693.

Yoon J-H, Prakash L, Prakash S. 2009. Highly error-free role of DNA polymerase $\eta$ in the replicative bypass of UV induced pyrimidine dimers in mouse and human cells. Proc Natl Acad Sci 106: 1821918224.

You Y-H, Pfeifer GP. 2001. Similarities in sunlight-induced mutational spectra of CpG-methylated transgenes and the p53 gene in skin cancer point to an important role of 5-methylcytosine rsidues in solar UV mutagenesis. J Mol Biol 305: 389-399.

You Y-H, Lee D-H, Yoon J-H, Nakajima S, Yasui A, Pfeifer GP. 2001. Cyclobutane pyrimidine dimers are responsible for the vast majority of mutations induced by UVB irradiation in mammalian cells. I Biol Chem 276: 44688-44694. 


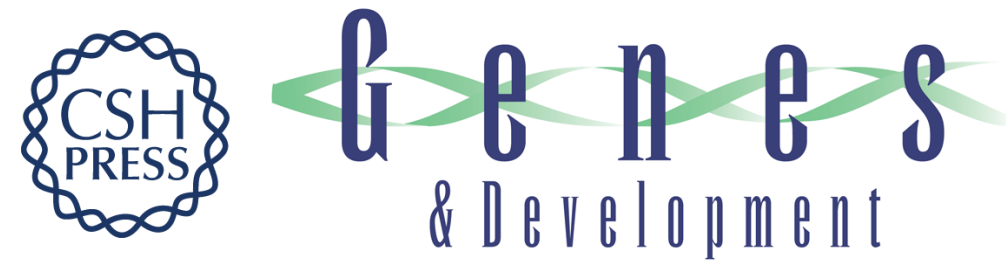

\section{Error-free replicative bypass of (6-4) photoproducts by DNA polymerase $\zeta$ in mouse and human cells}

Jung-Hoon Yoon, Louise Prakash and Satya Prakash

Genes Dev. 2010, 24:

Access the most recent version at doi:10.1101/gad.1872810

Supplemental http://genesdev.cshlp.org/content/suppl/2009/12/29/24.2.123.DC1
Material

References This article cites 20 articles, 11 of which can be accessed free at: http://genesdev.cshlp.org/content/24/2/123.full.html\#ref-list-1

License

Email Alerting

Receive free email alerts when new articles cite this article - sign up in the box at the top Service right corner of the article or click here.

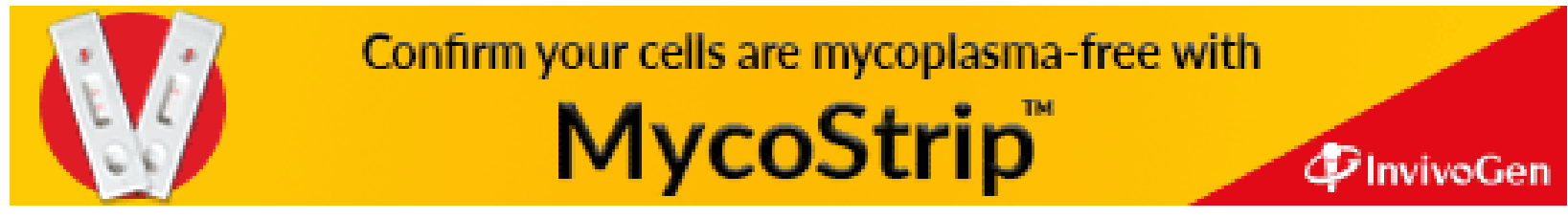

